\title{
Construction of Two-Dimensional Wavelength/Time Optical Orthogonal Codes Using Difference Family
}

\author{
Fong-Ray Gu and Jingshown Wu, Fellow, IEEE
}

\begin{abstract}
A new family of two-dimensional (2-D) wavelength/ time optical orthogonal codes (OOCs) for asynchronous optical code division multiple access (OCDMA) systems is proposed. The construction scheme uses the difference family (DF), which is an assemblage of difference sets in the combinatorial theory. It is proven that the proposed codewords satisfy the correlation properties required for the asynchronous OCDMA systems. The code dimension of the proposed codes is more flexible than that of the conventional 2-D codewords. The performance of the system with the proposed codes is analyzed by using the Markov-chain method. Numerical results show that the bit error rate (BER) has a minimal value given the number of simultaneous users. It is also observed that the maximum number of simultaneous users of the system can be achieved by properly choosing both the code weight and cross correlation of the 2-D OOCs.
\end{abstract}

Index Terms-Bit error rate (BER), code weight, difference family (DF), Markov chain, optical code division multiple access (OCDMA), optical orthogonal codes (OOCs).

\section{INTRODUCTION}

$\mathbf{C}$ ODE DIVISION multiple access (CDMA) has been well studied in the wireless communication systems. Recently, the spread-spectrum technique is getting a lot of attention in the optical fiber transmission due to the inherent large bandwidth of fibers. Optical CDMA (OCDMA) has several benefits such as asynchronous transmission, being flexible in network design, accommodation of burst traffic and variable bit-rate traffic, etc. Because of nonnegative power for optical signals, optical orthogonal codes (OOCs) are a family of $(0,1)$ sequences with good autocorrelation and cross-correlation properties. We denote $\left(n, w, \lambda_{\mathrm{a}}, \lambda_{\mathrm{c}}\right)$ OOCs as the OOCs with code length $n$. The off-peak autocorrelation $\lambda_{\mathrm{a}}$ and cross correlation $\lambda_{\mathrm{c}}$ are required to be small for accurate time synchronization and less multiple-user interference (MUI), respectively. The code weight $w$ should be large in order to distinguish the desired signal from the MUI and noise. If $\lambda_{\mathrm{a}}=\lambda_{\mathrm{c}}=\lambda$, the $\left(n, w, \lambda_{\mathrm{a}}, \lambda_{\mathrm{c}}\right)$ OOCs are simply denoted as $(n, w, \lambda)$ OOCs. The code size $\Phi$ is the total number of codewords of the $(n, w, \lambda)$ OOCs and can be written as [1]

$$
\Phi \leq\left\lfloor\frac{1}{w}\left\lfloor\frac{n-1}{w-1} \cdots\left\lfloor\frac{n-\lambda}{w-\lambda}\right\rfloor \ldots\right\rfloor\right\rfloor .
$$

Manuscript received January 6, 2004; revised May 25, 2005. Part of this work was supported by the National Science Council and the Ministry of Education, Taiwan, under Grant NSC91-2213-E-002-106 and Grant 89-E-FA06-2-4.

The authors are with the Department of Electrical Engineering and Graduate Institute of Communication Engineering, National Taiwan University, Taipei, Taiwan 10617, R.O.C.

Digital Object Identifier 10.1109/JLT.2005.855860
Larger code sizes can be obtained by relaxing the crosscorrelation constraint of the OOCs. For example, the code size of $(n, w, 1,2)$ OOCs is about ten times larger than that of $(n, w, 1)$ OOCs [2]. However, the bit error rate (BER) performance degrades as the cross correlation increases because of MUI. Therefore, there is a tradeoff between the BER and the code size. The performance bound of the OOCs with large cross correlation is found [3]. For a given code length and the number of simultaneous users, the BER performance of the OOCs has a minimal value when the code weight is optimal. Another way to improve the performance is to design a good receiver. The receiver with double optical hard limiters was discussed [4]. It is shown that the BER performance of the systems with double optical hard limiters is better than that without double optical hard limiters. Channel interference-cancellation techniques using interference estimation and double hard limiters were proposed and analyzed [5], [6]. The system with an interference-cancellation receiver can improve the error floor and accommodate more simultaneous users.

The $\left(n, w, \lambda_{\mathrm{a}}, \lambda_{\mathrm{c}}\right)$ OOCs spread the input data bits in the time domain and are called the one-dimensional (1-D) OOCs. The code length of the 1-D OOCs is always large in order to achieve good BER performance. However, long code sequences will occupy a large bandwidth and reduce the bandwidth utilization. Recently, the wavelength/time OOCs or the multiwavelength OOCs (MWOOCs), which encode the data bits in both wavelength and time domain, were proposed [7]. The MWOOCs are also called the two-dimensional (2-D) OOCs. The MWOOCs can support a larger number of cardinalities than 1-D OOCs using less time-spreading length. We denote $\left(m \times n, w, \lambda_{\mathrm{a}}, \lambda_{\mathrm{c}}\right)$ MWOOCs as the 2-D OOCs with $m$ wavelengths and time-spreading length $n$. The codeword of the MWOOCs can be considered as an $m \times n$ matrix with $(0,1)$ elements. The code weight $w$ is the number of 1's in the matrix. An example of two MWOOCs is shown in Fig. 1, where the shaded parts represent the code weight " 1 ."

Let $\delta$ represent the asynchronous time shift of the 2-D OCDMA systems. The autocorrelation and cross correlation of the MWOOCs have the following properties [7].

Definition 1: For any codeword $X=\left\{x_{i, j}\right\}$ of the $(m \times n$, $\left.w, \lambda_{\mathrm{a}}, \lambda_{\mathrm{c}}\right)$ MWOOCs with entry $x_{i j} \in\{0,1\}$, where $0 \leq i<m$ and $0 \leq j<n$, the periodic autocorrelation $\lambda_{\mathrm{a}}$ of $X$ satisfies

$$
\sum_{i=0}^{m-1} \sum_{j=0}^{n-1} x_{i, j} x_{i, j \oplus \delta} \leq \lambda_{\mathrm{a}}
$$

where $0<\delta<n$ and the subscripts $\oplus$ represents the modulo- $n$ addition. 

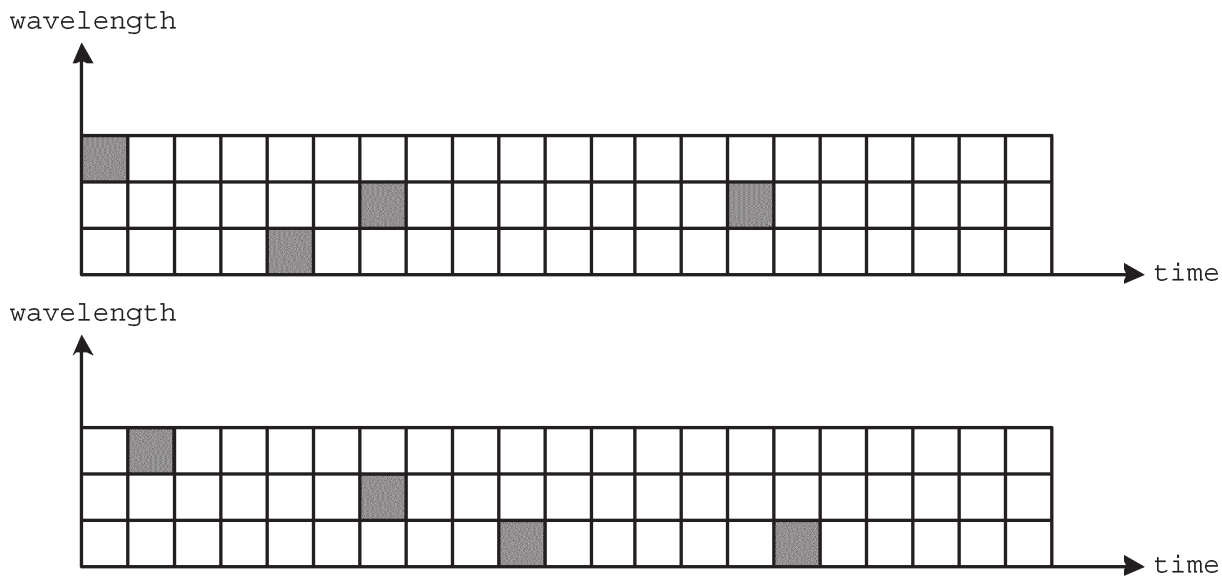

Fig. 1. Example of two $(3 \times 21,4,1,1)$ MWOOCs.

Definition 2: For any two codewords $X=\left\{x_{i, j}\right\}$ and $Y=$ $\left\{y_{i j}\right\}$ of the $\left(m \times n, w, \lambda_{\mathrm{a}}, \lambda_{\mathrm{c}}\right)$ MWOOCs with entries $x_{i j}$ and $y_{i j} \in\{0,1\}$, where $0 \leq i<m$ and $0 \leq j<n$, the periodic cross correlation $\lambda_{\mathrm{c}}$ of $X$ and $Y$ satisfies

$$
\sum_{i=0}^{m-1} \sum_{j=0}^{n-1} x_{i, j} y_{i, j \oplus \delta} \leq \lambda_{\mathrm{c}}
$$

where $0 \leq \delta<n$ and the subscripts $\oplus$ represents the modulo- $n$ addition.

Frequency-hopping sequences and conventional 1-D OOCs can be used to construct the MWOOCs [7]. However, the code dimensions of the conventional MWOOCs are restricted. It is observed that the spreading in the wavelength domain and the time domain are confined by certain relations, which are very stiff and inconvenient for designers. For example, the code dimension of the generalized multiwavelength Reed-Solomon codes (GMWRSC) is restricted as $(p \times(p-1) p)$, where $p$ is a prime number [7]. The MWOOCs derived from the 1-D OOCs have a code dimension of $(m \times m)$. In this paper, we propose a family of new MWOOCs. The construction scheme uses the difference family (DF), which is an assemblage of difference sets in the combinatorial theory. The proposed MWOOCs can have an arbitrary combination of the number of wavelengths $m$ and the number of chips $n$, where $m \leq n$.

This paper is organized as follows. We present the existence properties of the DF in Section II. The recursive construction, the construction of the DF from projective plane, and that from the affine plane are also described in this section. In Section III, the design of the new MWOOCs based on DF is given. In Section IV, the performance of the system with the proposed MWOOCs and double optical hard limiters is analyzed by using the Markov-chain method. Section V shows the numerical results. Finally, we give the conclusion in Section VI.

\section{DF IN GALOIS FIELDS (GFS)}

Let $G$ be an additive finite group of order $n$ and $S=$ $\left(B_{1}, B_{2}, \ldots, B_{s}\right)$ be a family of $s$ subsets (or base blocks), where $B_{\zeta}$ is the base block of $S$ for $\zeta=1,2, \ldots, s$. Each base block has size $k_{i}$ and $k_{i} \in K$, where $K=\left\{k_{1}, k_{2}, \ldots, k_{r}\right\}$ is a set of $r$ integers and $2 \leq k_{i} \leq n$ for $i=1,2, \ldots, r$. We denote $\Delta B_{\zeta}$ as a difference list of base block $B_{\zeta}$ and $\Delta B_{\zeta}=\left\{i-i^{\prime}(\bmod n) \mid i, i^{\prime} \in B_{\zeta}, i \neq i^{\prime}\right\}$ for $\zeta=1,2, \ldots, s$. If every entry of $\Delta B_{\zeta}$ has exactly $\lambda_{d}$ representations in $\Delta S=$ $\left(\Delta B_{1}, \Delta B_{2}, \ldots, \Delta B_{s}\right)$, then $S$ is an $\left(n, K, \lambda_{d}\right)$ DF [8]. When all the base blocks have the single size $k$, they form an $\left(n, k, \lambda_{d}\right)$ DF. A necessary condition of the $\left(n, k, \lambda_{d}\right) \mathrm{DF}$ is given as [8]

$$
\lambda_{d}(n-1) \equiv 0(\bmod (k(k-1))) .
$$

Some of the known existence statements of the $\left(n, k, \lambda_{d}\right)$ DF in GFs with $\lambda_{d}=1$ and $n<10000$ are presented in the following [8]-[12]. The DF outside this range is not discussed in this paper because it is impractical for the OCDMA systems.

Lemma 1: An $(n, 3,1) \mathrm{DF}$ in $\mathrm{GF}(n)$ always exists for any prime power $n=6 t+1$, where $t$ is a positive integer [8].

Lemma 2: For any prime power $n \equiv 1(\bmod 12)$, there exists an $(n, 4,1)$ DF in $\operatorname{GF}(n)$ [9].

Lemma 3: There exists an $(n, 5,1) \mathrm{DF}$ in $\mathrm{GF}(n)$ for any prime power $n \equiv 1(\bmod 20)$ [9].

Lemma 4: There exists an $(n, 6,1) \mathrm{DF}$ in $\mathrm{GF}(n)$ for any prime power $n \equiv 1(\bmod 30)$ with one exception of $n=61$ [10].

Lemma 5: If $n$ is a prime power, an $(n, 7,1) \mathrm{DF}$ in $\mathrm{GF}(n)$ exists when $n \equiv 1(\bmod 42)$ with exception of $n=43$ and possible exception of $n=127$, or $n=211$ [11].

Lemma 6: There exists an $(n, 8,1) \mathrm{DF}$ in $\operatorname{GF}(n)$ for any prime power $n \equiv 1(\bmod 56)$ with possible exception of $n=113, n=169, n=281$, or $n=337$ [12].

Lemma 7: There exists an $(n, 9,1) \mathrm{DF}$ in $\operatorname{GF}(n)$ for any prime power $n \equiv 1(\bmod 72)$ with possible exception of $n=289, n=361$, or $n=577$ [12].

The number of base blocks $s$ of the $(n, k, 1)$ DF can be obtained according to the following equation [8].

$$
(n-1)=s k(k-1) .
$$

If base block $B_{\zeta}$ has $k_{i}$ elements, then $B_{\zeta}=\left\{b_{\zeta 1}, b_{\zeta 2}, \ldots\right.$, $\left.b_{\zeta k_{i}}\right\}$. We denote the translation of $B_{\zeta}$ as $B_{\zeta}+l$ for any $l \in G$ and $B_{\zeta}+l=\left\{b_{\zeta 1}+l, b_{\zeta 2}+l, \ldots, b_{\zeta k_{i}}+l\right\}$. Two base blocks $B_{i}$ and $B_{j}$ are called equal if and only if they have the same element sets after the translation. In the DF, all base blocks are not equal. In the following sections, we will prove that the DF 
can be used to construct a family of codewords that have low off-peak autocorrelation and cross correlation.

\section{A. Properties of Pairwise Balanced Design (PBD)}

A PBD of order $v$ is a pair $(\psi, \beta)$, where $\psi$ is a set of $v$ elements and $B$ is a family of subsets (blocks). We assume that each block has size $k_{i}$ and $k_{i} \in K$, where $K=\left\{k_{1}, k_{2}, \ldots, k_{r}\right\}$ is a set of $r$ integers and $2 \leq k_{i} \leq v$ for $i=1,2, \ldots, r$. In the $(v, K, \lambda)$ PBD, every pair of distinct elements in $\psi$ occurs in exactly $\lambda$ blocks in $\beta$. When $\lambda=1$, the $(v, K, \lambda)$ PBD is usually denoted as $(v, K)$ PBD, which forms a linear space [13]. Thus, the blocks in the $(v, K)$ PBD are called lines. The balanced incomplete block design (BIBD) is a special case of $(v, k, \lambda)$ PBD with single block size $k$. Define $\alpha(K)=\operatorname{gcd}\{k-1$ : $k \in K\}$ and $\beta(K)=\operatorname{gcd}\{k(k-1): k \in K\}$, where $\operatorname{gcd}\{\cdot\}$ denotes the greatest common divisor.

Theorem 1: The necessary conditions for existence of a $(v, K) \mathrm{PBD}$ are [14]

$$
v-1=0(\bmod \alpha(K))
$$

and

$$
v(v-1)=0(\bmod \beta(K)) .
$$

We give some existence statements of the $(v, K)$ PBD for specified $K$ in the following.

Lemma 8: Let $q$ be a prime power. For any $0 \leq t \leq q+1$, there exists a $\left(q^{2}+q+1-t,\{q-1, q, q+1\}\right)$ PBD [8].

Lemma 9: Let $q$ be a prime power. For any $0 \leq t \leq q+1$, there exists a $\left(q^{2}-t,\{q-2, q-1, q\}\right)$ PBD [8].

Lemma 10: If $q$ is a prime power, for any $0 \leq t \leq$ $q+1$, there exists a $(q(q-1) / 2+t,\{(q-1) / 2,(q+1) / 2$, $(q+3) / 2\})$ PBD [15].

The upper bound of the number of blocks of the $(v, K)$ PBD is not known yet. However, the number of blocks of the $(v, K)$ $\mathrm{PBD}$ with $K=\{k\}$ is given as [16]

$$
s=\frac{v(v-1)}{k(k-1)} .
$$

\section{B. Construction of DF From Projective Plane Using PBD}

The projective geometry $\operatorname{PG}(d, q)$ is a vector space with elements from $\operatorname{GF}(q)$, where $q$ is a prime power and $d$ is a positive integer [17]. The $\operatorname{PG}(d, q)$ contains all subspaces of $(d+1)$-dimensional vector spaces. Two subspaces are equivalent if there exists $\gamma \in \operatorname{GF}(q)$ and $\gamma \neq 0$, such that $\bar{y}=\gamma \bar{x}$ for any two vector spaces $\bar{x}$ and $\bar{y}$ over $\operatorname{PG}(d, q)$. Therefore, the number of elements in $\mathrm{PG}(d, q)$ is $n=\left(q^{d+1}-1\right) /(q-1)$. If we take $d$-dimensional subspaces of $\operatorname{GF}(q)^{d+1}$, there are $v=\left(q^{d}-1\right) /(q-1)$ subspaces, which form a hyperplane. Thus, the number of hyperplanes is $\chi=\left(q^{d+1}-1\right) /(q-1)$. If every pair of distinct vectors of $d$-dimensional subspaces occurs in exactly $\lambda$ hyperplanes, then we have

$$
\lambda\left(\begin{array}{l}
n \\
2
\end{array}\right)=\chi\left(\begin{array}{l}
v \\
2
\end{array}\right)
$$

where $\left(\begin{array}{l}x \\ y\end{array}\right)$ is the number of combinations of $x$ elements taken $y$ at a time. From (9), we can obtain $\lambda=\left(q^{d-1}-1\right) /(q-1)$.

Theorem 2: The $\operatorname{PG}(d, q)$ is a block design with parameter $\left[\left(q^{d+1}-1\right) /(q-1),\left(q^{d}-1\right) /(q-1),\left(q^{d-1}-1\right) /(q-1)\right][8]$.

The point-hyperplane design of the $\operatorname{PG}(d, q)$ forms an $(n, v, \lambda)$ cyclic difference set with parameters $n=\left(q^{d+1}-\right.$ $1) /(q-1), v=\left(q^{d}-1\right) /(q-1)$, and $\lambda=\left(q^{d-1}-1\right) /(q-$ $1)$. It is also called the Singer difference set with classical parameters. Any pair of the elements $\left(i, i^{\prime}\right)$ has exactly $\lambda$ representations of the difference $i-i^{\prime}=\delta(\bmod n)$ for $i, i^{\prime} \in$ $\operatorname{PG}(d, q)$, and $i \neq i^{\prime}$. If $\lambda=1$, it is a $\left(q^{2}+q+1, q+1,1\right)$ Singer difference set and the $\operatorname{PG}(2, q)$ is called the projective plane [8]. The 2-D subspaces in $\operatorname{PG}(2, q)$ are called the lines and any two distinct lines intersect at one point.

The construction of DF from the projective plane is listed as the following.

1) First, construct the $\left(q^{2}+q+1, q+1\right.$, 1) Singer difference set from the projective plane $\operatorname{PG}(2, q)$. There are $q+1$ elements in the Singer difference set and the difference of any two elements occurs exactly once.

2) Pack the $q+1$ elements of the Singer difference set into blocks with size $k_{i}$, where $2 \leq k_{i} \leq q+1$ for $i=1,2, \ldots, r$. This is the $(v, K)$ PBD with parameters $v=q+1$ and $K=\left\{k_{1}, k_{2}, \ldots, k_{r}\right\}$

Then, we can obtain a family of blocks in which any difference $\delta\left(\bmod q^{2}+q+1\right)$ of the elements in a block occurs exactly once, i.e., an $\left(q^{2}+q+1, K, 1\right)$ DF. We give an example of the DF from the projective plane as the following.

Example 1: We consider the $(91,10,1)$ Singer difference set in $\operatorname{PG}(2,9)$ with elements $\{0,1,3,9,27,49,56,61,77,81\}$. Since $10=1(\bmod 3)$, then the $(10,(\{3,4\}))$ PBD exists. Therefore, we have the $(91,(\{3,4\}), 1) \mathrm{DF}$ as $\{1,27,61\},\{1,49,77\}$, $\{1,56,81\},\{3,27,81\}, \quad\{3,49,61\},\{3,56,77\},\{9,27,77\}$, $\{9,49,81\},\{9,56,61\},\{0,1,3,9\},\{0,27,49,56\},\{0,61,77,81\}$.

\section{Construction of DF From Affine Plane Using PBD}

The affine geometry $\operatorname{AG}(d, q)$ is a $d$-dimensional vector space over $\operatorname{GF}(q)$, where $d$ is a positive integer and $q$ is a prime power [17]. The number of elements in the $\operatorname{AG}(d, q)$ is $q^{d}$. If we take $(d-1)$-dimensional subspaces in an $\operatorname{AG}(d, q)$, then we can obtain a hyperplane that consists of $q^{d-1}$ subspaces. We first introduce the following theorem.

Theorem 3: Removing a block and all elements in the block from a symmetric $(n, v, \lambda)$ design yields an $(n-v, v-\lambda, \lambda)$ BIBD called a residual design [12].

The $\operatorname{AG}(d, q)$ can be constructed by removing a block and all elements in the block from the $\operatorname{PG}(d, q)$ [12]. In Theorem 2, we know that the $\operatorname{PG}(d, q)$ is a block design with parameter $\left[\left(q^{d+1}-1\right) /(q-1),\left(q^{d}-1\right) /(q-1),\left(q^{d-1}-1\right) /(q-1)\right]$. Therefore, the $\operatorname{AG}(d, q)$ is a residual design of a $\left[q^{d}, q^{d-1}\right.$, $\left.\left(q^{d-1}-1\right) /(q-1)\right]$ BIBD. From $(9)$, we can obtain the number of hyperplanes of $\mathrm{AG}(d, q)$ as $\left[\left(q^{d+1}-1\right) /(q-1)\right]-1$.

The point-hyperplane design of the $\operatorname{AG}(d, q)$ forms an $(n, v, \lambda)$ cyclic difference set with parameters $n=q^{d}, v=$ $q^{d-1}$, and $\lambda=\left(q^{d-1}-1\right) /(q-1)$ [17]. Any pair of the elements $\left(i, i^{\prime}\right)$ in the $q$ set of integers modulo $q^{2}$ has exactly one 
representation of the difference $i-i^{\prime}=\delta\left(\bmod q^{2}\right)$ for any residue $\delta \neq 0\left(\bmod q^{2}\right)$. If $\lambda=1$, the $\operatorname{AG}(2, q)$ is called the affine plane [8]. The 2-D subspaces in the $\operatorname{AG}(2, q)$ are called the lines and any two distinct lines intersect at only one point.

The construction of DF from the affine plane is listed as the following.

1) First, construct the $\left(q^{2}, q, 1\right)$ cyclic difference set from the affine plane. There are $q$ elements in the cyclic difference set and the difference of any two elements occurs exactly once.

2) Pack the $q$ elements of the cyclic difference set into blocks with size $k_{i}$, where $2 \leq k_{i} \leq q$ for $i=1,2, \ldots, r$. This is the $(v, K)$ PBD with parameters $v=q$ and $K=\left\{k_{1}, k_{2}, \ldots, k_{r}\right\}$.

Then, we can obtain a family of blocks in which any difference $\delta\left(\bmod q^{2}\right)$ of the elements in a block occurs exactly once, i.e., a $\left(q^{2}, K, 1\right) \mathrm{DF}$.

\section{Recursive Construction of DF}

We present here two recursive construction schemes of DF. First, we can construct a new DF from two DFs according to the following lemma.

Lemma 11: If there are $\left(n_{1}, k, \lambda_{1}\right) \mathrm{DF}$ in $\mathrm{GF}\left(n_{1}\right)$ and $\left(n_{2}, k, \lambda_{2}\right)$ DF in $\operatorname{GF}\left(n_{2}\right)$, then an $\left(n_{1} n_{2}, k, \lambda_{1} \lambda_{2}\right)$ DF in $\mathrm{GF}\left(n_{1} n_{2}\right)$ exists [12].

Second, when an $(n, k, \lambda)$ DF in $\mathrm{GF}(n)$ exists, we can construct a new DF by using this $(n, k, \lambda) \mathrm{DF}$ according to the following lemma.

Lemma 12: If there is an $(n, k, \lambda) \mathrm{DF}$ in $\operatorname{GF}(n)$, then an $\left(n^{a}, k, \lambda\right) \mathrm{DF}$ exists in $\mathrm{GF}\left(n^{a}\right)$ for any positive integer $a[18]$.

\section{Design OF MWOOCs Using DF AND THEIR CORRELATION PROPERTIES}

In this section, we present the design procedure of the proposed $\left(m \times n, w, 1, \lambda_{c}\right)$ MWOOCs using DF with single block size $k$. The design procedures using the DF with various block sizes are similar. The procedure is described as follows.

1) Let the number of base blocks $s$ be larger than or equal to the number of wavelengths $m$. From (5), the value of $k$ can be determined. Then, we can construct an $(n, k, 1)$ DF as discussed in the previous section.

2) Place all base blocks of the $(n, k, 1)$ DF in rows of a matrix. We can obtain an $m \times k$ matrix and denote the matrix as the base matrix $B$.
3) Since each row of the base matrix has $k$ elements, there are $m k$ elements in the base matrix. If the desired code weight is $w$, we then partition the $m k$ elements of the base matrix into a family of subsets. Each subset has $w$ elements and any two subsets have $\lambda_{c}$ elements in common.

4) Cyclic shift the rows of each subsets vertically and we can obtain $m$ subsets. Then, check the cross correlation between the subsets of the shifted version and the others. Discard the subset if the cross correlation is larger than $\lambda_{\mathrm{c}}$.

5) The elements in the subset represent the mark positions of an $m \times n$ matrix and other entries of the matrix are " 0 ." Then, the $m \times n$ matrix is a codeword of the proposed $\left(m \times n, w, 1, \lambda_{\mathrm{c}}\right)$ MWOOCs.

According to the definition of DF, no $(n, k, 1)$ DF exists when $k=1$. However, $k=1$ is the special case of the construction procedure above. It represents that the base matrix $B$ contains elements from the additive finite group of order $n$ and each row has only one element. We should not shift the rows of the base matrix $B$ vertically when $k=1$. This is because the shifted matrix is the translation of $B$ and is therefore identical to $B$. An example is shown to construct the proposed $\left(m \times n, w, 1, \lambda_{\mathrm{c}}\right)$ MWOOCs.

Example 2: We show a construction of $(5 \times 21,4,1,1)$ MWOOCs as the following.

First, we construct the base blocks of the $(21,2,1)$ DF as: $\{0,1\},\{4,14\},\{0,16\},\{1,14\},\{14,16\},\{0,4\},\{0,14\}$, $\{1,4\},\{1,16\},\{4,16\}$. Then, one of the base matrices can be written as

$$
B=\left[\begin{array}{cc}
0 & 1 \\
4 & 14 \\
0 & 16 \\
1 & 14 \\
4 & 16
\end{array}\right]
$$

Next, we partition the ten elements of $B$ into a family of subsets. Each subset contains four elements and any two subsets have one element in common. A subset with elements that represent the mark positions of the codeword is shown at the bottom of the page.

The family of $(5 \times 21,4,1,1)$ MWOOCs from the base matrix $B$ is listed in Table I.

Theorem 4: The off-peak autocorrelation of the proposed $\left(m \times n, w, 1, \lambda_{c}\right)$ MWOOCs is at most 1.

Proof: Let $X=\left\{x_{i j}\right\}$ be a codeword of the $(m \times$ $\left.n, w, 1, \lambda_{c}\right)$ MWOOCs and $x_{i j} \in\{0,1\}$, for $0 \leq i<m$ and

$$
\left[\begin{array}{ll}
0 & \\
& \\
0 & 16 \\
1 &
\end{array}\right] \Longrightarrow\left[\begin{array}{lllllllllllllllllllll}
1 & 0 & 0 & 0 & 0 & 0 & 0 & 0 & 0 & 0 & 0 & 0 & 0 & 0 & 0 & 0 & 0 & 0 & 0 & 0 & 0 \\
0 & 0 & 0 & 0 & 0 & 0 & 0 & 0 & 0 & 0 & 0 & 0 & 0 & 0 & 0 & 0 & 0 & 0 & 0 & 0 & 0 \\
1 & 0 & 0 & 0 & 0 & 0 & 0 & 0 & 0 & 0 & 0 & 0 & 0 & 0 & 0 & 0 & 1 & 0 & 0 & 0 & 0 \\
0 & 1 & 0 & 0 & 0 & 0 & 0 & 0 & 0 & 0 & 0 & 0 & 0 & 0 & 0 & 0 & 0 & 0 & 0 & 0 & 0 \\
0 & 0 & 0 & 0 & 0 & 0 & 0 & 0 & 0 & 0 & 0 & 0 & 0 & 0 & 0 & 0 & 0 & 0 & 0 & 0 & 0
\end{array}\right]
$$


TABLE I

FAMILY OF $(5 \times 21,4,1,1)$ MWOOCS FROM THE BASE MATRIX $B$ IN EXAMPLE 2

\begin{tabular}{|c|c|}
\hline & 1100000000000000000000000 \\
\hline & 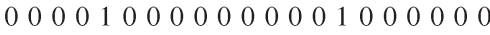 \\
\hline \multirow[t]{5}{*}{$\mathrm{C}_{1}$} & 000000000000000000000000 \\
\hline & 00000000000000000000000 \\
\hline & 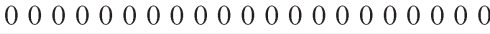 \\
\hline & 100000000000000000000000000 \\
\hline & 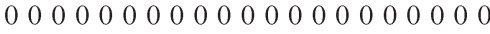 \\
\hline \multirow[t]{5}{*}{$\mathrm{C}_{2}$} & 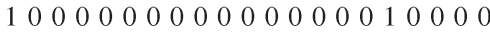 \\
\hline & 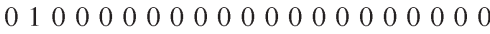 \\
\hline & 0000000000000000000000 \\
\hline & 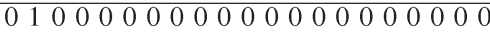 \\
\hline & $\begin{array}{llllllllllll}0 & 0 & 0 & 0 & 0 & 0 & 0 & 0 & 0 & 0 & 0\end{array}$ \\
\hline \multirow[t]{5}{*}{$\mathrm{C}_{3}$} & 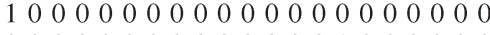 \\
\hline & 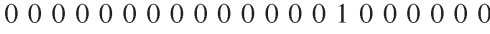 \\
\hline & 000001000000000000000000000 \\
\hline & 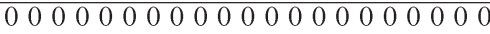 \\
\hline & 0000010000000000000000000 \\
\hline \multirow[t]{5}{*}{$\mathrm{C}_{4}$} & 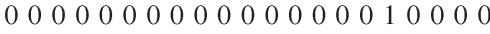 \\
\hline & 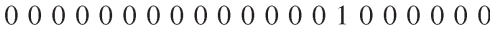 \\
\hline & 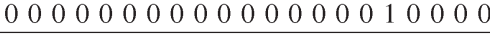 \\
\hline & $\begin{array}{llllllllllllllllllllllllllll} & 0 & 0 & 0 & 0 & 0 & 0 & 0 & 0 & 0 & 0 & 0 & 0 & 0 & 0 & 0 & 0 & 0 & 0 & 0\end{array}$ \\
\hline & 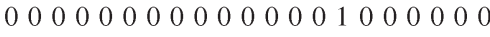 \\
\hline \multirow[t]{3}{*}{$\mathrm{C}_{5}$} & 00000000000000000000000 \\
\hline & 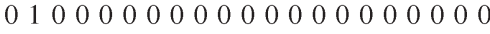 \\
\hline & 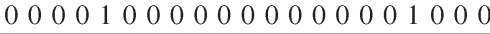 \\
\hline
\end{tabular}

$0 \leq j<n$. When there exists a time shift $\delta$ and $\delta \neq 0$, the offpeak autocorrelation of $X$ can be written as

$$
\begin{aligned}
\sum_{i=0}^{m-1} \sum_{j=0}^{n-1} x_{i, j} x_{i, j \oplus \delta}= & \sum_{j=0}^{n-1} x_{0, j} x_{0, j \oplus \delta}+\sum_{j=0}^{n-1} x_{1, j} x_{1, j \oplus \delta} \\
& +\cdots+\sum_{j=0}^{n-1} x_{(m-1), j} x_{(m-1), j \oplus \delta} \\
= & \lambda_{\mathrm{a} 0}+\lambda_{\mathrm{a} 1}+\cdots+\lambda_{\mathrm{a}(m-1)} .
\end{aligned}
$$

Since the codeword is constructed from the $(n, k, 1) \mathrm{DF}$, the difference of any two mark positions occurs exactly once. If $\lambda_{\mathrm{a} t}=1$ for $t \in\{0,(m-1)\}$, it is impossible to have an integer $t^{\prime} \in\{0,(m-1)\}, t^{\prime} \neq t$ such that $\sum_{j=0}^{n-1} x_{t^{\prime}, j} x_{t^{\prime}, j \oplus \delta}=1$, i.e., $\lambda_{\mathrm{a} t^{\prime}}=0$. Therefore, the off-peak autocorrelation of the MWOOCs is at most 1 .

Theorem 5: The cross correlation of the proposed $(m \times n$, $\left.w, 1, \lambda_{c}\right)$ MWOOCs is at most $\lambda_{c}$.

Proof: Let $X=\left\{x_{i j}\right\}$ and $Y=\left\{y_{i j}\right\}$ be two codewords of the $\left(m \times n, w, 1, \lambda_{c}\right)$ MWOOCs and $x_{i j}, y_{i j} \in\{0,1\}$, for $0 \leq i<m$ and $0 \leq j<n$. When there exists a time shift $\delta$ between the two codewords, the cross correlation of $X$ and $Y$ can be written as

$$
\begin{aligned}
\sum_{i=0}^{m-1} \sum_{j=0}^{n-1} x_{i, j} y_{i, j \oplus \delta}= & \sum_{j=0}^{n-1} x_{0, j} y_{0, j \oplus \delta}+\sum_{j=0}^{n-1} x_{1, j} y_{1, j \oplus \delta} \\
& +\cdots+\sum_{j=0}^{n-1} x_{(m-1), j} y_{(m-1), j \oplus \delta} \\
= & \lambda_{\mathrm{c} 0}+\lambda_{\mathrm{c} 1}+\cdots+\lambda_{\mathrm{c}(m-1)} .
\end{aligned}
$$

If $\delta=0$, the two codewords intersect at $\lambda_{\mathrm{c}}$ elements since they are constructed from the $\left(m k, w, \lambda_{c}\right)$ design. If $\delta \neq 0$, then $\lambda_{c t} \leq 1$ for $t \in\{0,(m-1)\}$ because the difference of any two mark positions occurs exactly once. We assume that $X$ and $Y$ have more than one hits when $\delta \neq 0$. Then, in (11), $\lambda_{\mathrm{c} t}=1$ appears more than once for $t \in\{0,(m-1)\}$. This contradicts the property of the $(n, k, 1) \mathrm{DF}$. Therefore, the cross correlation of the MWOOCs is 0,1 , or $\lambda_{c}$.

The properties of the $(n, k, 1)$ DF make the off-peak correlation of the MWOOCs not greater than 1 . The $\left(m k, w, \lambda_{\mathrm{c}}\right)$ design guarantees the cross correlation of any two codewords less than or equal to $\lambda_{c}$. Therefore, a set of $\left(m \times n, w, 1, \lambda_{c}\right)$ MWOOCs can be constructed by the proposed design procedure. On the other hand, according to the construction procedure, the proposed codewords have flexible code dimension. It is very desirable for designing the MWOOCs in the multiwavelength OCDMA systems.

Theorem 6: The $t-(v, k, \varsigma)$ packing design is a collection of subsets from $\psi$, where $\psi$ is a set of $v$ elements, such that every $t$ distinct element occurs in at most $\varsigma$ subsets [19]. Denote $D_{\varsigma}(v, k, t)$ as the maximum number of subsets in any $t-(v, k, \varsigma)$ packing design and is given as

$$
\left.D_{\varsigma}(v, k, t) \leq\left\lfloor\frac{v}{k}\left\lfloor\frac{v-1}{k-1}\left\lfloor\cdots\left\lfloor\frac{v-t+1}{k-t+1} \varsigma\right\rfloor\right\rfloor\right\rfloor\right\rfloor\right\rfloor .
$$

Theorem 7: The upper bound of the number of subsets obtained from the base block $B$ is

$$
\begin{aligned}
D \leq\left\lfloor\frac { v ( v - 1 ) } { w ( k - 1 ) } \left\lfloor\frac{v(v-1)-(k-1)}{(w-1)(k-1)}\right.\right. \\
\left.\left.\times\left\lfloor\ldots\left\lfloor\frac{v(v-1)-\lambda_{c}(k-1)}{\left(w-\lambda_{c}\right)(k-1)}\right\rfloor \ldots\right\rfloor\right\rfloor\right\rfloor
\end{aligned}
$$

where $v=q+1$ and $v=q$ for the DF from $\operatorname{PG}(2, q)$ and $\operatorname{AG}(2, q)$, respectively, and $k$ is obtained from

$$
m=\frac{v(v-1)}{k(k-1)}
$$

Proof: Assume that the number of wavelengths $m$ is equal to the number of available base blocks $s$. It would not lose the accuracy when deriving the upper bound of $D$. Then, the following equation holds:

$$
m=s=\frac{v(v-1)}{k(k-1)}
$$

where $v=q+1$ and $v=q$ for the DF from projective plane $\operatorname{PG}(2, q)$ and affine plane $\operatorname{AG}(2, q)$, respectively. The number of elements in the base block $B$ is $m k=v(v-1) /(k-1)$. If the desired code weight is $w$, we partition the $m k$ elements of $B$ into a family of subsets with $w$ elements, and any two subsets have $\lambda_{c}$ elements in common. This procedure is a $\left(\lambda_{\mathrm{c}}+1\right)-(m k, w, 1)$ packing design. Therefore, the number 


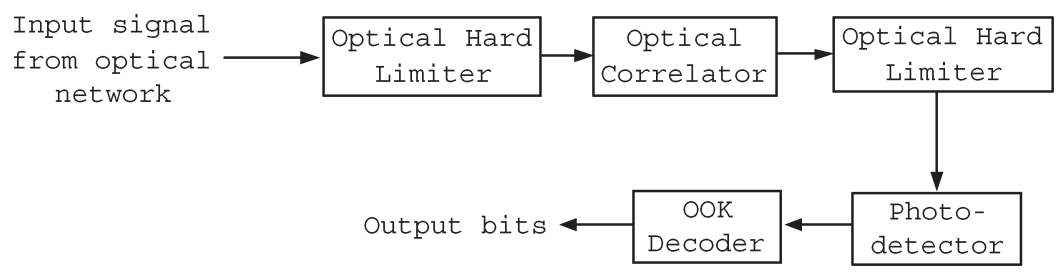

Fig. 2. Receiver structure of asynchronous OCDMA systems using double optical hard limiters.

of subsets from the base block $B$ can be obtained according to Theorem 6 as

$$
\begin{aligned}
D \leq\left\lfloor\frac{m k}{w}\left\lfloor\frac{m k-1}{w-1}\left\lfloor\ldots\left\lfloor\frac{m k-\lambda_{\mathrm{c}}}{w-\lambda_{\mathrm{c}}}\right\rfloor \ldots\right\rfloor\right\rfloor\right\rfloor \\
=\left\lfloor\frac { v ( v - 1 ) } { w ( k - 1 ) } \left\lfloor\frac{v(v-1)-(k-1)}{(w-1)(k-1)}\right.\right. \\
\left.\quad \times\left\lfloor\cdots\left\lfloor\frac{v(v-1)-\lambda_{\mathrm{c}}(k-1)}{\left(w-\lambda_{\mathrm{c}}\right)(k-1)}\right\rfloor \ldots\right\rfloor\right\rfloor .
\end{aligned}
$$

\section{Performance Analysis of the Systems With THE PROPOSED MWOOCS}

We analyze the BER performance with consideration of shot noise, thermal noise, APD bulk, and surface leakage currents. The receiver structure is shown in Fig. 2 [4]. We assume that the system is chip synchronous among users since it is the worst case of the performance. The average photon arrival rate $\varepsilon$ per pulse is given by

$$
\varepsilon=\frac{\eta P_{W}}{h f}
$$

where $\eta$ is the APD quantum efficiency, $P_{W}$ is the received signal power, $h$ is the Planck's constant, and $f$ is the optical frequency. The optical signal power after the second hard limiter will be limited to two levels: "ON" and "OFF" levels. We denote state $S_{1}$ as the optical signal power of the "ON" level and state $S_{0}$ of the "OFF" level. The average photon arrival rate is $\varepsilon$ for state $S_{1}$, otherwise the photon arrival rate is 0 . For state $S_{b}, b \in\{0,1\}$, the probability density function (pdf) of the output current $\Psi_{b}$ of the photodetector is assumed Gaussian and given by [20]

$$
P_{\Psi_{b}}(\varphi)=\frac{1}{\sqrt{2 \pi \sigma_{b}^{2}}} e^{-\frac{\left(\varphi-\mu_{b}\right)^{2}}{2 \sigma_{b}^{2}}}
$$

where $\mu_{b}$ is the mean value of the photodetector output current given by

$$
\mu_{b}=G T_{\mathrm{c}}\left(b \varepsilon+\frac{I_{\mathrm{b}}}{e}\right)+\frac{T_{\mathrm{c}} I_{\mathrm{s}}}{e}
$$

and $G$ is the average APD gain, $T_{\mathrm{c}}$ is the chip time, $e$ is the electron charge, $I_{\mathrm{b}} / e$ is the contribution of the APD bulk leakage current to the APD output, and $I_{\mathrm{S}}$ is the APD surface leakage current. The variance of the photo current $\sigma_{b}^{2}$ can be expressed as

$$
\sigma_{b}^{2}=G^{2} F_{\mathrm{e}} T_{\mathrm{c}}\left(b \varepsilon+\frac{I_{\mathrm{b}}}{e}\right)+\frac{T_{\mathrm{c}} I_{\mathrm{s}}}{e}+\sigma_{\mathrm{th}}^{2}
$$

where $F_{\mathrm{e}}$ is the excess noise factor given by

$$
F_{\mathrm{e}}=k_{\mathrm{eff}} G+\left(2-\frac{1}{G}\right)\left(1-k_{\mathrm{eff}}\right)
$$

where $k_{\text {eff }}$ is the APD effective ionization ratio, and $\sigma_{\text {th }}^{2}$ is the variance of thermal noise expressed as

$$
\sigma_{\mathrm{th}}^{2}=\frac{2 k_{\mathrm{B}} T_{\mathrm{r}} T_{\mathrm{c}}}{e^{2} R_{\mathrm{L}}}
$$

where $k_{\mathrm{B}}$ is the Boltzmann's constant, $T_{\mathrm{r}}$ is the receiver noise temperature, and $R_{\mathrm{L}}$ is the receiver load resistance.

In order to minimize the error probability, we set the threshold of the decision circuit $\theta$ as

$$
\theta=\frac{\mu_{0} \sigma_{1}+\mu_{1} \sigma_{0}}{\sigma_{1}+\sigma_{0}}
$$

If the output current $\Psi_{b}$ is larger than $\theta$, the output data bit $b_{\mathrm{o}}$ is decided to be bit one, or otherwise bit zero. The probability that the state $S_{b}$ is decoded erroneously due to photodetector noise is given by

$$
\operatorname{Pr}\left(b_{\mathrm{o}} \neq S_{b}\right)=\frac{1}{2} \operatorname{erfc}\left(\frac{\mu_{b}-\theta}{\sqrt{2 \sigma_{b}^{2}}}\right)
$$

where $\operatorname{erfc}(\cdot)$ is the complementary error function expressed as

$$
\operatorname{erfc}(z)=\frac{2}{\sqrt{\pi}} \int_{z}^{\infty} \exp \left(-u^{2}\right) \mathrm{d} u
$$

We assume that the desired signal uses $\left(m \times n, w, 1, \lambda_{\mathrm{c}}\right)$ MWOOCs of codeword $X$ and the interfering signal uses the codeword $Y$, where $X=\left\{x_{i j}\right\}$ and $Y=\left\{y_{i j}\right\}$ for $0 \leq i \leq$ $m-1$ and $0 \leq j \leq n-1$. The number of interfered marks of the desired codeword can be expressed as

$$
I_{\delta}=\sum_{i=0}^{m-1} \sum_{j=0}^{n-1} x_{i, j} y_{i, j \oplus \delta}
$$

where $\delta$ is the asynchronous time shift. The probability that one of the code weights of $X$ hit by $Y$ is $w / 2 m n$, where the factor $1 / 2$ represents that each user transmits data 0 and 1 with equal 


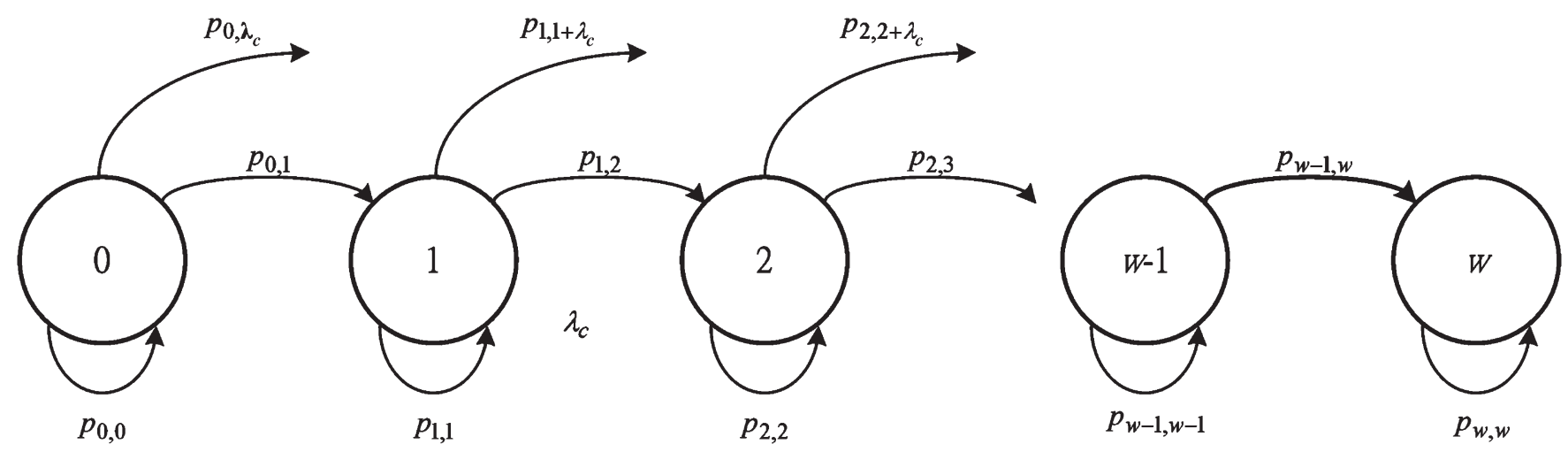

Fig. 3. Model of Markov chain.

probability. Since there are $w$ code weights in the codeword $X$, then the expected value of $I_{\delta}$ is given by

$$
E\left[I_{\delta}\right]=w \cdot \frac{w}{2 m n} .
$$

Let $q_{1}$ and $q_{\lambda_{c}}$ be the probabilities that the desired signal is hit by the interfering user at one mark and $\lambda_{\mathrm{c}}$ marks, respectively. Thus, the expected value of $I_{\delta}$ can also be expressed as

$$
E\left[I_{\delta}\right]=q_{1}+\lambda_{\mathrm{c}} \cdot q_{\lambda_{\mathrm{c}}}
$$

where $q_{\lambda_{c}}=1 / 2 m n$. From (26) and (27), we can obtain $q_{1}$ as

$$
q_{1}=\frac{w^{2}-\lambda_{\mathrm{c}}}{2 m n} .
$$

We model the number of code weights interfered by $\vartheta$ interfering users as the states of the Markov chain, as shown in Fig. 3 [21]. The state transition occurs when a new interfering user appears in the system, i.e., $\vartheta \rightarrow \vartheta+1$. Then, the statetransition probability $p_{i, j}$ can be written as

$$
p_{i, j}= \begin{cases}q_{0}+q_{1} \frac{i}{w}+q_{\lambda_{\mathrm{c}}} \frac{\left(\begin{array}{c}
i \\
\lambda_{c}
\end{array}\right)}{\left(\begin{array}{c}
w \\
\lambda_{c}
\end{array}\right)}, & \text { if } j=i \\
q_{1} \frac{w-i}{w}+q_{\lambda_{c}} \frac{\left(\begin{array}{c}
i c \\
\lambda_{c}-1
\end{array}\right)\left(\begin{array}{c}
w-i \\
1
\end{array}\right)}{\left(\begin{array}{c}
w \\
\lambda_{c}
\end{array}\right)}, & \text { if } j=i+1 \\
q_{\lambda_{\mathrm{c}}} \frac{\left(\begin{array}{c}
w-i \\
\lambda_{c}
\end{array}\right)}{\left(\begin{array}{c}
w \\
\lambda_{c}
\end{array}\right)}, & \text { if } j=i+\lambda_{c} \\
0, & \text { otherwise }\end{cases}
$$

where $q_{0}=1-q_{1}-q_{\lambda_{c}}$. Let $\kappa^{(\vartheta)}=\left[\kappa_{0}^{(\vartheta)}, \kappa_{1}^{(\vartheta)}, \ldots, \kappa_{w}^{(\vartheta)}\right]$ be a $1 \times(w+1)$ vector and represent the state probability of the Markov chain given $\vartheta$ interfering users. If the state-transition matrix is $\mathbf{P}$ where $\mathbf{P}=\left\{p_{i, j}\right\}$ for $0 \leq i, j \leq w$, the following relation holds:

$$
\kappa^{(\vartheta)}=\kappa^{(\vartheta-1)} \mathbf{P}
$$

The state probability $\kappa^{(\vartheta)}$ can be obtained recursively as $\kappa^{(\vartheta)}=$ $\kappa^{(0)} \mathbf{P}^{\vartheta}$, where $\kappa^{(0)}$ is the initial state of the Markov chain. Since $\kappa^{(0)}$ represents no interfering user in the system, then $\kappa^{(0)}=[1,0, \ldots, 0]$. For the decoder with double hard limiters, the signal state at the second hard limiter output will be $S_{1}$ if the transmitted data bit $b$ is 1 . If the transmitted data bit is 0 , the second hard limiter cannot entirely remove the MUI when the number of interfering marks exceeds or equals the
TABLE II SYSTEM PARAMETERS

\begin{tabular}{llll}
\hline \hline Name & Symbol & value & unit \\
\hline light wavelength & & 1.3 & $\mu m$ \\
APD quantum efficiency & $\eta$ & 0.6 & \\
APD gain & $G$ & 100 & \\
APD effective ionization ratio & $k_{e f f}$ & 0.02 & \\
APD bulk leakage current & $I_{b}$ & 0.1 & $\mathrm{nA}$ \\
APD surface leakage current & $I_{s}$ & 10 & $\mathrm{nA}$ \\
chip duration & $T_{c}$ & 0.1 & $\mathrm{~ns}$ \\
bit rate & $R_{b}=\frac{1}{n T_{c}}$ & & \\
receiver noise temperature & $T_{r}$ & 300 & $K$ \\
receiver load resistor & $R_{L}$ & 1030 & $\Omega$ \\
\hline
\end{tabular}

code weight. Therefore, the probability that an error occurs at the output of the second hard limiter is equal to the probability that the Markov chain is at state $w$. If there are $N$ simultaneous users in the OCDMA system, we have

$$
\begin{aligned}
P_{\mathrm{e}} & =\operatorname{Pr}\left(S_{1} \mid b=0\right) \\
& =\kappa_{w}^{(N-1)} .
\end{aligned}
$$

The total BER is given as

$$
\begin{aligned}
\mathrm{BER}= & \operatorname{Pr}\left(b_{o}=1 \mid S_{0}, b=0\right) \operatorname{Pr}\left(S_{0} \mid b=0\right) \operatorname{Pr}(b=0) \\
& +\operatorname{Pr}\left(b_{o}=1 \mid S_{1}, b=0\right) \operatorname{Pr}\left(S_{1} \mid b=0\right) \operatorname{Pr}(b=0) \\
& +\operatorname{Pr}\left(b_{o}=0 \mid S_{0}, b=1\right) \operatorname{Pr}\left(S_{0} \mid b=1\right) \operatorname{Pr}(b=1) \\
& +\operatorname{Pr}\left(b_{o}=0 \mid S_{1}, b=1\right) \operatorname{Pr}\left(S_{1} \mid b=1\right) \operatorname{Pr}(b=1) \\
= & \frac{1}{2} \operatorname{erfc}\left(\frac{\theta-\mu_{0}}{\sqrt{2 \sigma_{0}^{2}}}\right) \operatorname{Pr}\left(S_{0} \mid b=0\right)+\frac{1}{2} \operatorname{erfc}\left(\frac{\mu_{1}-\theta}{\sqrt{2 \sigma_{1}^{2}}}\right) \\
& +\frac{1}{2}\left(1-\operatorname{erfc}\left(\frac{\mu_{1}-\theta}{\sqrt{2 \sigma_{1}^{2}}}\right)\right) \operatorname{Pr}\left(S_{1} \mid b=0\right)
\end{aligned}
$$

where $\operatorname{Pr}\left(S_{0} \mid b=0\right)=1-\operatorname{Pr}\left(S_{1} \mid b=0\right)$.

\section{NUMERICAL RESUlTS}

The system parameters are listed in Table II [2]. Unlike the advantage of the proposed codewords, the conventional 


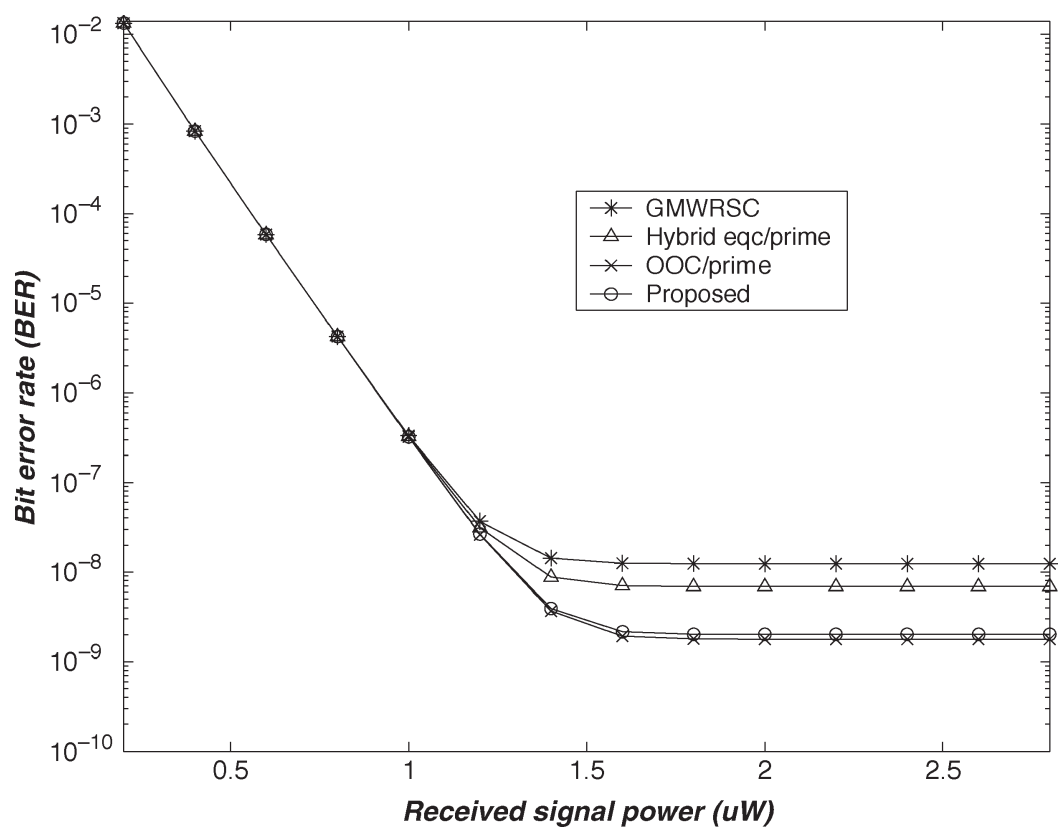

Fig. 4. BER performance versus the received optical signal power of the $(13 \times 13(13-1), 13,1,1)$ GMWRSC, the $(11 \times 11(2 \cdot 11-1))$ hybrid eqc/prime MWOOCs, the $(13 \times 183,1,1)$ OOC/prime MWOOCs, and the proposed $(13 \times 183,13,1,1)$ MWOOCs when the number of simultaneous users is 100 .

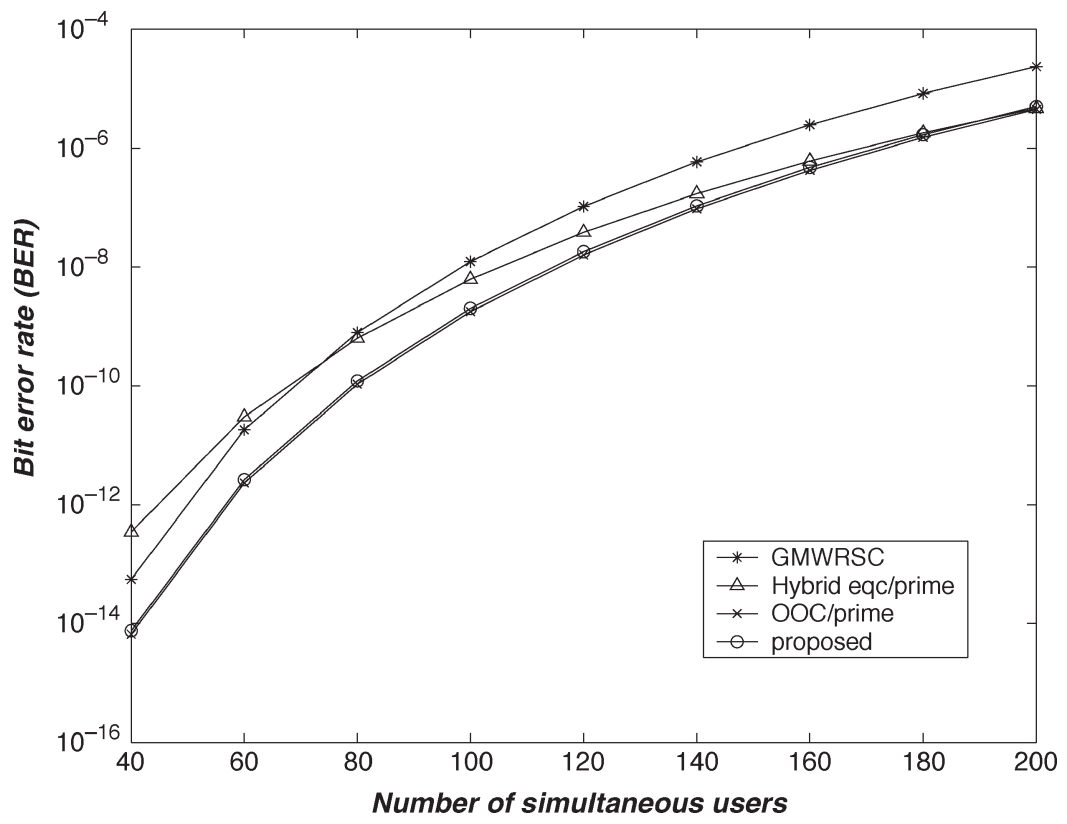

Fig. 5. BER performance versus the number of simultaneous users of the $(13 \times 13(13-1), 13,1,1)$ GMWRSC, the $(11 \times 11(2 \cdot 11-1))$ hybrid eqc/prime MWOOCs, the $(13 \times 183,1,1)$ OOC/prime MWOOCs, and the proposed $(13 \times 183,13,1,1)$ MWOOCs when the received optical signal power is $2.2 \mu$ W.

MWOOCs have fixed code dimension. In order to compare the proposed codes with the others, we assume that the codewords are with approximately the same code dimension. Fig. 4 shows the BER performance versus the received optical signal power of the $(13 \times 13(13-1), 13,1,1)$ GMWRSC [7], the $(11 \times 11(2 \cdot 11-1))$ hybrid extended quadratic congruence codes $(\mathrm{eqc}) /$ prime MWOOCs [22], the $(13 \times 183,1,1)$ OOC/prime MWOOCs [23], and the proposed $(13 \times 183,13$, $1,1)$ MWOOCs when the number of simultaneous users is 100. Fig. 5 illustrates the BER performance versus the number of simultaneous users when the received optical signal power is $2.2 \mu \mathrm{W}$. Numerical results show that the GMWRSC and the hybrid eqc/prime MWOOCs have higher BER than that of the proposed code and the OOC/prime MWOOCs. This is because the stiff code dimension of GMWRSC increases the hit probability, while the large cross correlation for hybrid eqc/prime codes induces large MUI. The BER performance of the proposed MWOOCs is nearly identical to that of the OOC/prime MWOOCs because both of the codewords have the same correlation constraint.

Fig. 6 shows the BER of the proposed $\left(m \times n, 10,1, \lambda_{c}\right)$ MWOOCs from $\operatorname{PG}(2,13)$ and $\operatorname{AG}(2,13)$ versus the received 


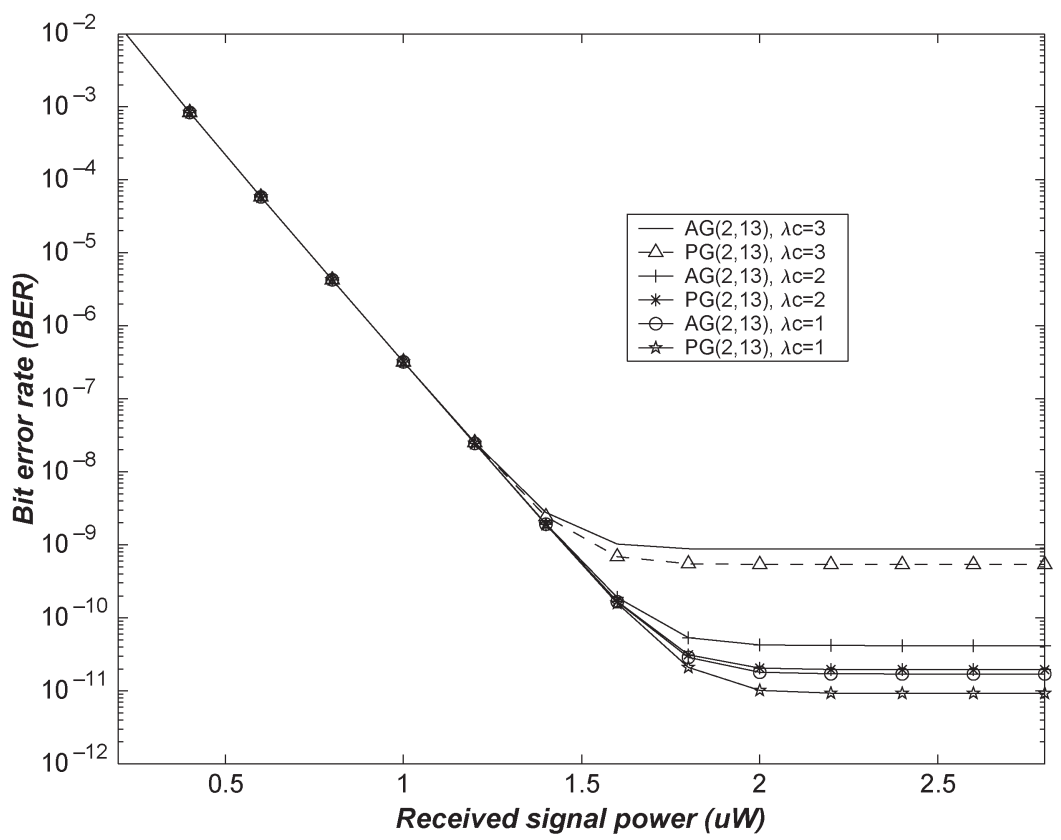

Fig. 6. BER of the $\left(m \times n, 10,1, \lambda_{\mathrm{c}}\right)$ MWOOCs versus received optical signal power when $m=30$ and $n=183$ and 169 for PG(2,13) and AG(2,13), respectively. The number of simultaneous users $N$ is 100 .

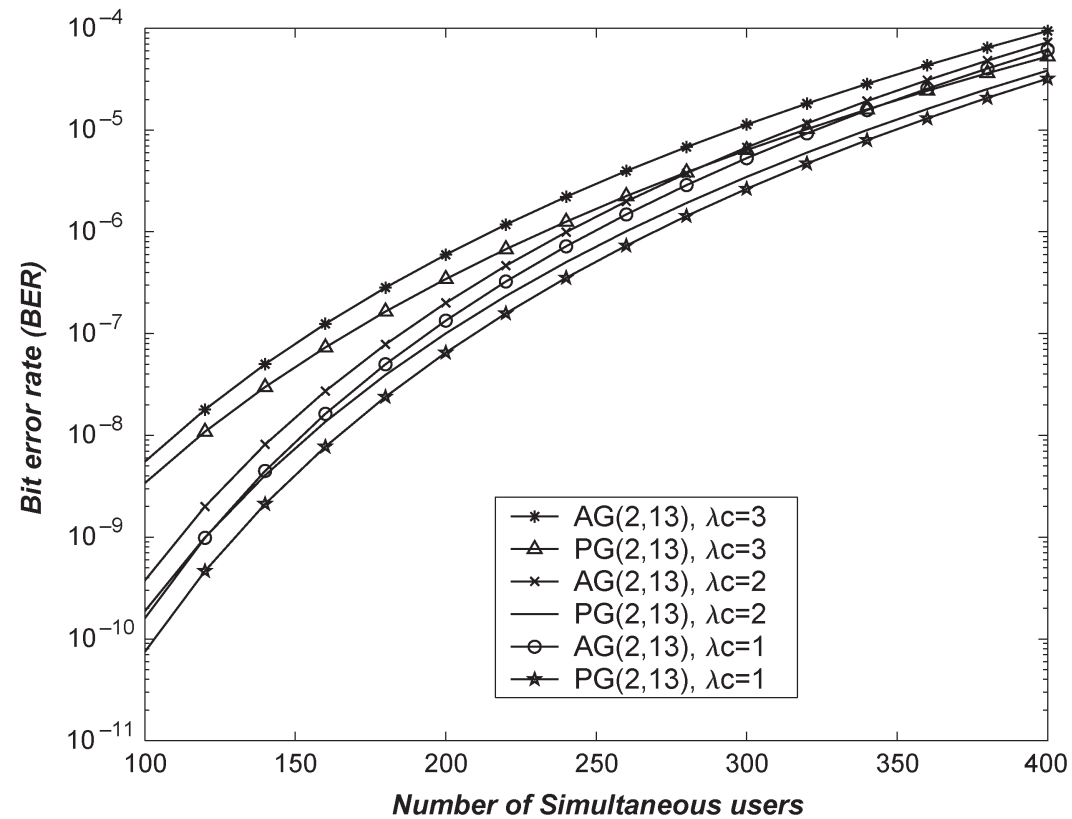

Fig. 7. BER performance of the $\left(m \times n, 10,1, \lambda_{\mathrm{C}}\right)$ MWOOCs versus the number of simultaneous users when $m=30$ and $n=183$ and 169 for PG(2,13) and $\mathrm{AG}(2,13)$, respectively. The received optical signal power is $2.2 \mu \mathrm{W}$.

optical signal power when the number of wavelengths $m=30$ and the number of chips in the time domain $n=183$ and 169 for $\operatorname{PG}(2,13)$ and $\operatorname{AG}(2,13)$, respectively. The BER decreases as the received signal power increases. There is an error floor due to MUI. Meanwhile, a large value of cross correlation of the MWOOCs degrades the system performance. Fig. 7 shows the BER of the proposed $\left(m \times n, 10,1, \lambda_{\mathrm{c}}\right)$ MWOOCs versus the number of simultaneous users when $m=30$ and $n=183$ and 169 for $\mathrm{PG}(2,13)$ and $\mathrm{AG}(2,13)$, respectively. The received optical signal power is $2.2 \mu \mathrm{W}$. The BER rises when the number of simultaneous users increases. The performance of the codewords constructed from $\mathrm{PG}(2,13)$ is superior to that from $\mathrm{AG}(2,13)$, because $\mathrm{PG}(2,13)$ constructs codewords with larger code dimension and smaller average MUI than that of $\operatorname{AG}(2,13)$.

Fig. 8 shows the BER of the proposed $\left(m \times n, w, 1, \lambda_{c}\right)$ MWOOCs versus the code weight $w$ when $m=30$ and $n=183$ and 169 for $\operatorname{PG}(2,13)$ and $\mathrm{AG}(2,13)$, respectively. The received optical signal power is $2.2 \mu \mathrm{W}$ and the number of simultaneous users is 250 . When the code weight is small, the BER decreases as the code weight increases. The BER achieves a minimal value at the optimum code weights and 


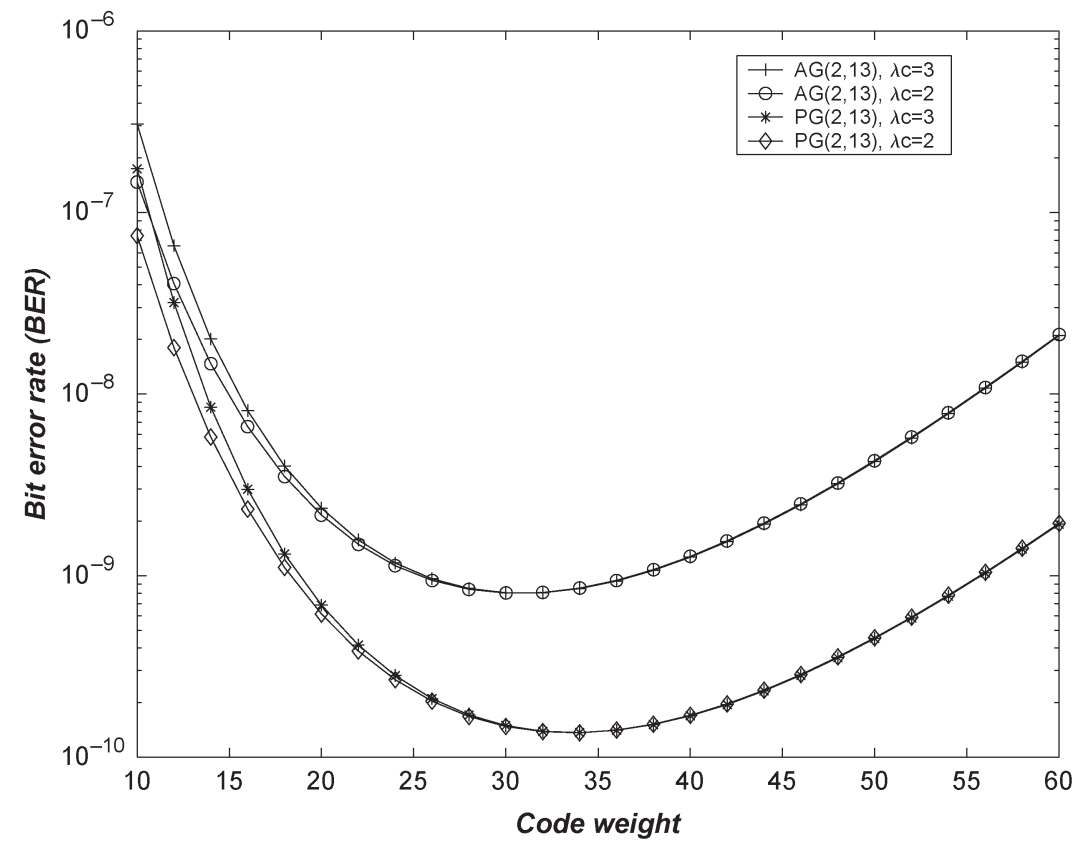

Fig. 8. BER performance of the $\left(m \times n, w, 1, \lambda_{\mathrm{c}}\right)$ MWOOCs versus the code weight $w$ when $m=30$ and $n=183$ and 169 for PG(2,13) and AG(2,13), respectively. The received optical signal power is $2.2 \mu \mathrm{W}$, and the number of simultaneous users $N$ is 250 .

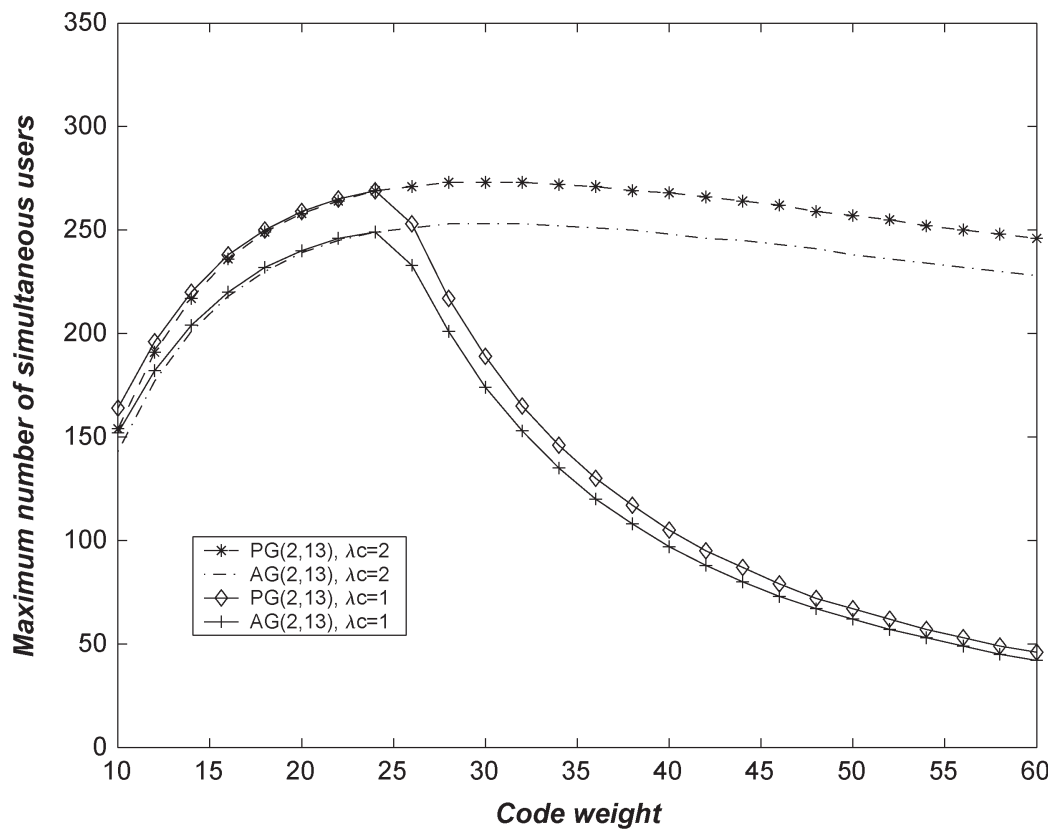

Fig. 9. Number of simultaneous users of the $\left(m \times n, w, 1, \lambda_{\mathrm{c}}\right)$ MWOOCs versus the code weight $w$ when $m=30$ and $n=183$ and 169 for PG(2,13) and $\mathrm{AG}(2,13)$, respectively. The received optical signal power is $2.2 \mu \mathrm{W}$.

then rises while the code weight increases. This is because the interfering users with large code weight will induce more MUI. Fig. 9 shows the number of simultaneous users of the proposed $\left(m \times n, w, 1, \lambda_{\mathrm{c}}\right)$ MWOOCs versus the code weight at BER $\leq$ $10^{-9}$. We observe that the number of simultaneous users of the MWOOCs has a maximal value. The number of simultaneous users is small for small code weight. When the code weight is getting large, according to Fig. 8, the BER has a minimal value and therefore, the number of simultaneous users of the system is upper bounded. Note that for $\lambda_{\mathrm{c}}=1$, the number of simultaneous users decreases significantly with increasing code weight. This is because the number of simultaneous users of the MWOOCs, with $\lambda_{\mathrm{c}}=1$, is limited by the code size.

\section{CONCLUSION}

We construct a family of 2-D wavelength/time OOCs by using the DF. Three construction schemes of the DF-the PBD construction from the projective plane, the PBD construction from the affine plane, and the recursive construction-are given in this paper. We place the base blocks of the DF in rows of the base matrix. The family of MWOOCs is constructed, for 
the first time, from the base matrix. The proposed MWOOCs satisfy the correlation properties required for the asynchronous OCDMA systems. The flexible code dimension of the proposed MWOOCs makes the codewords very desirable in the multiwavelength OCDMA systems. We analyze the system performance with double optical hard limiters by using the Markov-chain method. Numerical results show that the proposed MWOOCs have better BER performance than that of the GMWRSC and hybrid eqc/prime MWOOCs under approximately the same code dimension. The BER of the proposed MWOOCs is nearly identical to that of the OOC/prime MWOOCs because both of the codewords have the same correlation constraint. We show the minimal BER of the proposed MWOOCs at the optimum code weights. The maximal number of simultaneous users versus the code weight of the proposed MWOOCs at BER $\leq 10^{-9}$ is also illustrated. The number of simultaneous users of the codewords with $\lambda_{\mathrm{c}}=1$ is limited by the code size and decreases significantly. Therefore, both the code weight and cross correlation of the MWOOCs should be considered in designing the OCDMA systems with maximal capacity.

\section{REFERENCES}

[1] G. C. Yang and T. E. Fuja, "Optical orthogonal codes with unequal autoand cross-correlation constraints," IEEE Trans. Inf. Theory, vol. 41, no. 1, pp. 96-106, Jan. 1995.

[2] C. S. Weng and J. Wu, "Optical orthogonal codes with nonideal cross correlation," J. Lightw. Technol., vol. 19, no. 12, pp. 1856-1863, Dec. 2001.

[3] — " "Optical orthogonal codes with large crosscorrelation and their performance bound for asynchronous optical CDMA systems," J. Lightw. Technol., vol. 21, no. 3, pp. 735-742, Mar. 2003.

[4] T. Ohtsuki, "Performance analysis of direct-detection optical asynchronous CDMA systems with double optical hard-limiters," J. Lightw. Technol., vol. 15, no. 3, pp. 452-457, Mar. 1997.

[5] C. L. Lin and J. Wu, "A synchronous fiber-optic CDMA system using adaptive optical hardlimiters," J. Lightw. Technol., vol. 16, no. 8, pp. 1393-1403, Aug. 1998.

[6] K. Wakafuji and T. Ohtsuki, "Direct-detection optical CDMA receiver with interference estimation and double optical hardlimiters," J. Lightw. Technol., vol. 21, no. 10, pp. 2182-2188, Oct. 2003.

[7] G. C. Yang and W. C. Kwong, "Performance comparison of multiwavelength CDMA and WDMA+CDMA for fiber-optic networks," IEEE Trans. Commun., vol. 45, no. 11, pp. 1426-1434, Nov. 1997.
[8] T. Beth, D. Jungnickel, and H. Lenz, Design Theory. Cambridge, U.K.: Cambridge Univ. Press, 1999.

[9] M. Buratti, "Constructions for $(q, k, 1)$ difference families with $q$ a prime power and $k=4,5$," Discrete Math., vol. 138, no. 1-3, pp. 169-175, Mar. 1995.

[10] K. Chen and L. Zhu, "Existence of $(q, 6,1)$ difference families with $q$ a prime power," Designs Codes Cryptogr., vol. 15, no. 2, pp. 167-173, Nov. 1998.

[11] K. Chen, R. Wei, and L. Zhu, "Existence of $(q, 7,1)$ difference families with $q$ a prime power," J. Comb. Des., vol. 10, no. 2, pp. 126-138, Mar. 2002.

[12] C. J. Colbourn and J. H. Dinitz, CRC Handbook of Combinatorial Designs. Boca Raton, FL: CRC, 1996.

[13] M. Buratti, "Pairwise balanced designs from finite fields," Discrete Math., vol. 208-209, pp. 103-117, 1999.

[14] R. M. Wilson, "An existence theory for pairwise balanced designs II. The structure of PBD-closed sets and the existence conjectures," J. Comb. Theory, Ser. A, vol. 13, no. 2, pp. 246-273, 1972.

[15] A. C. H. Ling, X. Zhu, C. J. Colbourn, and R. C. Mullin, "Pairwise balanced designs with consecutive block sizes," Designs Codes Cryptogr., vol. 10, no. 2, pp. 203-222, Feb. 1997.

[16] P. Erdös and H. Hanani, "On a limit theorem in combinatorial analysis," Publ. Math. Debr., vol. 10, pp. 10-13, 1963.

[17] A. Pott, Finite Geometry and Character Theory. New York: SpringerVerlag, 1995.

[18] R. M. Wilson, "Cyclotomy and difference families in elementary abelian groups," J. Number Theory, vol. 4, no. 1, pp. 17-47, Feb. 1972.

[19] R. Fuji-Hara and Y. Miao, "Optical orthogonal codes: Their bounds and new optimal constructions," IEEE Trans. Inf. Theory, vol. 46, no. 7 , pp. 2396-2406, Nov. 2000.

[20] H. M. Kwon, "Optical orthogonal code-division multiple-access system-Part I: APD noise and thermal noise," IEEE Trans. Commun., vol. 42, no. 7, pp. 2470-2479, Jul. 1994.

[21] J. J. Chen and G. C. Yang, "CDMA fiber-optic systems with optical hard limiters,” J. Lightw. Technol., vol. 19, no. 7, pp. 950-958, Jul. 2001.

[22] L. Tančevski and I. Andonovic, "Hybrid wavelength hopping/time spreading schemes for use in massive optical networks with increased security," J. Lightw. Technol., vol. 14, no. 12, pp. 2636-2647, Dec. 1996.

[23] W. C. Kwong, G. C. Yang, V. Baby, C. S. Brès, and P. R. Prucnal, "Multiple-wavelength optical orthogonal codes under prime-sequence permutations for optical CDMA," IEEE Trans. Commun., vol. 53, no. 1, pp. 117-123, Jan. 2005.

Fong-Ray Gu, photograph and biography not available at the time of publication.

Jingshown Wu (S'73-M'78-SM'99-F'05), photograph and biography not available at the time of publication. 